\title{
13 | El Japonismo en Marion Mahony Griffin y Walter Burley Griffin. De los conceptos gráficos del Ukiyo-e hacia una estrategia proyectual alternativa Japanism in Marion Mahony Griffin and Walter Burley Griffin. From Ukiyo-e graphical concepts to an alternative design strategy _Javier Mosquera González
}

\section{Introducción}

La arquitectura realizada por Marion Mahony Griffin y Walter Burley Griffin durante las tres primeras décadas del siglo XX en tres continentes diferentes, América, Oceanía y Asia, ha de considerarse como mestiza debido a la condición itinerante de sus autores [1]. Situados de forma deliberada al margen de los movimientos arquitectónicos predominantes entonces, el carácter ecléctico de sus obras reside en la libre reinterpretación de modelos arquitectónicos históricos, aplicados a las realidades locales especificas adaptadas a las condiciones naturales del entorno en el que son construidos.

"La cultura japonesa muestra un método de estudio en la práctica del dibujo en la enseñanza, en lugar de hacer lo que los europeos hacen. Estos tratan de dibujar directamente de los modelos con la idea de copiarlos. Los japoneses hacen su propio estudio del modelo, tanto si es una planta o un animal, y dibujan después desde su memoria, no desde el modelo en sí mismo, esto es, extrayendo los elementos que desean para sus propósitos, y sin incorporar muchas de las características irrelevantes que se muestran al hacer un dibujo de un objeto... esto es para mí el uso racional del arte, no una simple reproducción fotográfica" ${ }^{1}$. Del análisis de sus obras y su método de representación gráfico característico se desprende una influencia oriental, común entre otros arquitectos contemporáneos, pero que en su caso consigue trascender los límites del dibujo hasta convertirse en una filosofía inherente a la concepción de sus proyectos ${ }^{2}$.

\section{Una nueva sensibilidad}

Educados en el seno de familias cercanas al movimiento de la Iglesia Unitaria, la concepción monoteísta de esta, que revela el interés en el desarrollo del hombre como ser ligado únicamente a la Tierra, será una de las bases teóricas sobre las que ambos soportarán gran parte de sus obras como arquitectos. El hombre es dueño de su destino y, como tal, ha de vivir en comunión con la naturaleza. Su afinidad con los pensadores trascendentalistas, que reclaman una relación entre hombre y su entorno que trascienda los límites físicos ${ }^{3}$, resulta una evolución de las ideas unitarias, concediendo un papel fundamental al individuo como base para una sociedad alternativa. Solo así el ser humano entenderá que forma parte de un todo mayor, el universo, y que a su vez todos los seres que en él habitan se encuentran dentro de su persona.

Desde la observación de la naturaleza y las leyes que la configuran, el hombre será capaz de entender su papel en relación con esta al descubrir que el desarrollo personal del individuo implica un contacto directo con el entorno natural. El pensamiento de Edward Carpenter ${ }^{4}, y$ su visión del hombre como un ser salvaje que debe volver a la naturaleza, reforzará el deseo de Mahony y Griffin de convertir en realidad estos pensamientos. Descubren en la arquitectura, y especialmente en la figura del arquitecto como individuo, a alguien que puede materializar estos principios y por tanto, con la capacidad para educar a la sociedad desde la obra construida. El respeto por la naturaleza y la precisión con la que esta es representada en sus documentos gráficos, se debe a su interés por la arquitectura del paisaje. Esta debe entenderse como un proceso en el que el resultado final deviene de una concepción teórica y sensorial que busca un modo de vida en comunión con su entorno, hasta entonces poco explorada por el pensamiento occidental.

La cultura japonesa y sus métodos de representación, mostrados por primera vez en Estados Unidos en la Exposición Universal de Chicago de 1893 [2], atraerá a ambos con intensidad. Fundamentada en un profundo respeto hacia la naturaleza y el entorno en el que se inserta la arquitectura, encontrarán en ella un referente desde el continente asiático que reforzará muchas de sus convicciones personales. El Japonismo, entendido como la influencia que el arte oriental ejerce sobre el occidental desde la aplicación de técnicas y principios compositivos del primero, se convierte entonces en una fuente de inspiración para artistas plásticos deseosos de encontrar alternativas a los modelos conocidos ${ }^{5}$. La influencia en movimientos artísticos como el Impresionismo sirve como referente para establecer una nueva relación entre el individuo y su entorno, capaz de representar más allá de la realidad visible. El carácter absoluto del
Resumen pág 16 | Bibliografía pág 24

Universidad Politécnica de Madrid. Tulane University.

Arquitecto, Master en Proyectos Arquitectónicos Avanzados y Doctorando por la ETSAM en la UPM. Ponente en varios congresos internacionales de arquitectura como la UIA 2017 World Architects Congress en Seúl o el Foro de Arquitectura y Sociedad en la Universidad de Anáhuac de México. Ayudante docente en el departamento de proyectos de la ETSAM y Tulane Design Teaching Fellow, en la Tulane School of Architecture.

jmg@mosqueragonzalez.com

\section{Palabras clave}

Naturaleza, Marion Mahony Griffin, Walter Burley Griffin, Ukiyo-e, Rock Crest / Rock Glen, Castlecrag, efímero Nature, Marion Mahony Griffin, Walter Burley Griffin, Ukiyo-e, Rock Crest / Rock Glen, Castlecrag, ephimeral

Método de financiación Tulane Design Teaching Fellow en la Tulane School of Architecture, en colaboración con la Escuela Técnica Superior de Arquitectura de Madrid.

\section{GRIFFIN, Walter Burley. "Architecture in American Universities", Journal of Proceed- ings of Royal Victorian Institute of Architects, Septiembre 1913, pp. 170-184.}

[1] Marion Mahony Griffin y Walter Burley Griffin, Castlecrag, 1930. Fuente: Biblioteca Nacional de Australia.

[2] Pabellón de Japón Feria Internacional de Chicago. 1893. Fuente: Colección digital de la Biblioteca Paul V. Galvin.

[3] Lámina n 35 de ejercicios. Fuente: Arthu W. Dow. Composition. A series of exercises selected from a new system of education.

[1]

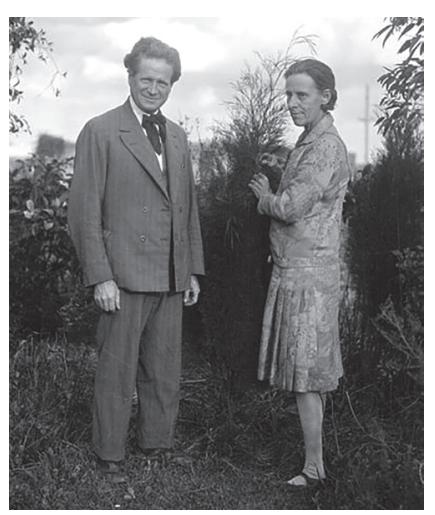


2 La caracterización de ambos como arquitectos en los que la relación entre el paisaje $y$ las propuestas planteadas se convierte en signo distintivo de sus obras dentro del panorama general de la Escuela de Chicago, es desarrollado en profundidad en $\mathrm{PEISCH}$, Mark. The Chicago School of Architecture: Early Followers of Sullivan and Wright. Nueva York: Random House, 1964. Asimismo narra el periodo formativo junto a Frank Lloyd Wright en el que desarrollan los proyectos para las Prairie Houses, al tiempo que presenta el interés

general de la sociedad americana por la cultura asiática, en particular la nipona y su método de representación gráfico. Las referencias a las influencias orientales en el sistema gráfico de Mahony y Griffin han sido estudiadas previamente por diversos autores como Anna Rubbo, ofreciendo una visión general de las técnicas empleadas derivadas de las utilizadas en Asia, o Christopher Vernon incidiendo en la relevancia del paisaje en la concepción general de sus proyectos, ambos en V.V.A.A. Beyond Architecture. Marion Mahony Griffin and Walter Burley Griffin. America. Australia. India. Sydney: Powerhouse publishing, 1998; pp. 40-55 y pp 86-103. Los artículos recopilados en WOOD, Deborah. Marion Mahony Griffin. Drawing the Form of Nature. Evanston, Illinois: Mary and Leigh Block Museum of Art, 2005. El trabajo de investigación de TINCH, Rebecca. Locating Modernity: Japonisme, Gender, and Enchantment at the 1893 World's Fair. Tesis fin de Máster. Directora: Mary E. Frederickson. Miami University, 2012, establece el marco histórico sobre el que trazar las relaciones entre oriente $y$ occidente, y la influencia del Japonismo, desde la perspectiva de Mahony y su relación con la práctica profesional en el estudio de Wright. Asimismo la relación específica con el Ukiyo-e y la representación gráfica ha sido analizada en ROBERTS, Ellen. "Ukiyo-e in Chicago: Frank Lloyd Wright, Marion Mahony Griffin and the Prairie School". Art in Print, Vol. 3, n'. 2, Julio Agosto 2013, pp. 3-10, atendiendo únicament a cuestiones compositivas y gráficas tras comparar los dibujos orientales con los realizados por Mahony y Griffin. Estos documentos sirven como referentes para plantear una nueva interpretación de la obra de estos autores, en la que los conceptos derivados de la representación de la naturaleza y la relación entre lo efimero y lo esencial, propios del pensamiento oriental, son aplicados como herramientas proyectuales en sus obras.

${ }^{3}$ Considera al individuo como aquel capaz de generar una reforma de la sociedad en la que vive, desde el profundo conocimiento de si mismo en relación con su entorno. La ética natural según la que pretenden restablecer el equilibrio entre el ser humano y la naturaleza precisa de un contacto directo con ella, en una especie de reencuentro con el origen de ambos. Las figuras de Ralph Waldo Emerson y Henry David Thoreau se convierten en referentes para Mahony y Griffin, debido a su carácter revolucionario desde la conciencia de la naturaleza como aliada para descubrir modelos sociales alternativos.

${ }^{4}$ CARPENTER, Edward. Civilization: Its Cause and Cure, and Other Essays. Londres: G. Allen \& Unwin, 1914.

${ }^{5}$ V.V.A.A. Japonismo. La fascinación por el arte japonés. Barcelona: Obra Social La Caixa, 2013, pp. 34-60.

[3]
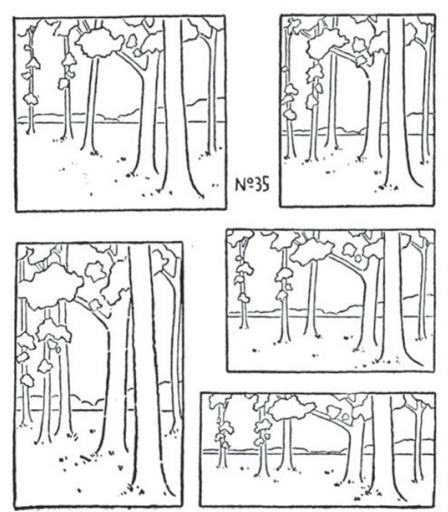

pensamiento oriental en el que cada elemento que rodea al ser humano ha de ser observado y valorado con el mismo respeto al formar parte de un todo superior, es algo que atrae a los arquitectos norteamericanos como alternativa para redefinir un modelo social en crisis.

Pasadas apenas dos décadas desde el final de la Guerra de Secesión Americana, y en una transición social del modelo productivo rural al urbano industrial, esta comunión con la naturaleza es interpretada como signo inequívoco de que tras esa filosofía pueden definirse los nuevos principios sobre los que crear un nuevo estilo arquitectónico de un país que necesita refundarse. Del interés creciente por las técnicas que definen el estilo gráfico japonés deviene el desarrollo de diferentes textos divulgativos, entre los que destacan el libro publicado en 1886 por Edward Morse ${ }^{6}$, en el que analiza diferentes obras niponas y sus métodos compositivos, y el manual redactado en 1899 por el artista americano Arthur W. Dow ${ }^{7}$ [3], quien recoge una teoría del arte basada en la interpretación del arte oriental, en particular atendiendo a su composición, las sombras y el color.

Conocedores de estos textos en su etapa como estudiantes de arquitectura, tras finalizar su formación académica y trabajar como colaboradores en el edificio Steinway Hall en Chicago, Mahony y Griffin se unen al equipo de trabajo de Wright ${ }^{8}$. Todos ellos encuentran en el arte nipón una fuente de inspiración para desarrollar una arquitectura propia del siglo XX, alejada de los modelos clásicos que no representaban al nuevo modelo social que debía definirse en el país estadounidense. Siendo Wright un gran conocedor y entusiasta de la cultura nipona, además de un gran coleccionista de ejemplares de estampas japonesas recopiladas tras diversos viajes al país asiático, la estancia en su estudio de una persona tan dotada como Mahony para la representación gráfica, influyó intensamente sobre este y la manera de dibujar sus proyectos.

La invitación que recibe Wright para presentar su trabajo en Europa, en la ciudad de Berlín, supone una oportunidad para consolidar ese nuevo modelo gráfico iniciado con Mahony. El contenido del portfolio editado por la Wasmuth Verlag será dibujado prácticamente en su totalidad por ella, incluyendo proyectos anteriores a la presencia de esta junto a Wright, desde 1893 hasta 1909. Como él mismo reconoce en una de las anotaciones realizadas en una de las láminas para la casa K. C. De Rhodes en 1906, "dibujado por Mahony, después de F. LL. W. e Hiroshige" [4]. Será la primera vez que el sistema de representación desarrollado por esta se muestre al público europeo. El esfuerzo de síntesis que supone la creación de este documento coincide con el pensamiento de Wright y el de sus colaboradores, con respecto a la eliminación de lo superfluo en la arquitectura, y con aquello que reconoce que ha percibido que ocurre en la arquitectura japonesa que le interesa ${ }^{9}$. En 1912 , Wright publica un ensayo sobre arte japonés ${ }^{10}$ en el que establece algunos de los principios que más tarde definirán su arquitectura orgánica. El texto incide en las ideas fundamentales de un estilo gráfico capaz de condensar la belleza universal de todo aquello que los artistas nipones representan. No solo descubre un nuevo método de trabajo basado en la geometría básica interna de cada elemento dibujado, sino una forma de ser en el mundo alternativa. El método gráfico iniciado entonces, e influenciado por el manual de Arthur. H. Dow, se había convertido en una herramienta mucho más precisa capaz de contar no solo la naturaleza y las especies vegetales locales, sino la nueva arquitectura propuesta ${ }^{11}$.

El valor simbólico implícito en la representación oriental ofrece a los arquitectos una herramienta capaz de generar imágenes evocadoras, realidades no construidas pero que contienen valores universales reconocibles por los espectadores, que esconden a su vez principios estructurales fundamentales ${ }^{10}$. El sistema gráfico oriental ensalza la belleza intrínseca de cada elemento representado no por el hecho de ser un objeto específico sino por el carácter propio de cada

[2]

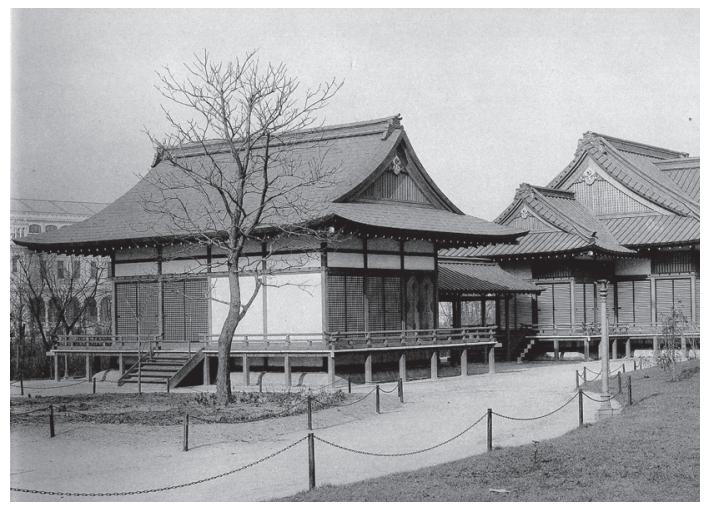

[4]

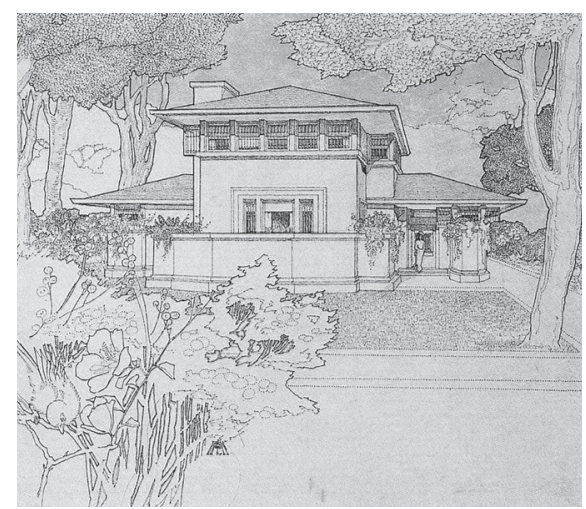


uno de ellos de forma universal. La percepción de lo inmediato, desde la representación occidental próxima al realismo, descubre en las técnicas orientales una dimensión capaz de mostrar lo atemporal, aquello que verdaderamente significa un objeto determinado, hasta convertirlo en algo común para todo aquel que lo observe. No se trata de representar el árbol sino el concepto de este. Además, cada una de las partes que lo componen contiene en sí mismas la cualidad de ser árbol.

La condición sensorial del arte japonés, en el que se enfatiza la relación entre hombre y naturaleza, se sirve de la intuición del espectador y del artista para, mediante la abstracción, expresar un significado a través de la representación de un objeto determinado. Será esta la característica que Mahony y Griffin apliquen a sus dibujos y que estará presente en las ideas básicas contenidas en cada uno de sus proyectos. Tratan de mostrar escenas posibles de objetos no construidos, generando en el espectador la sensación de pertenencia a un mundo ideal, pero a su vez capaz de transmitir serenidad y armonía en quien lo observa. Mahony describe la arquitectura del paisaje como la "consideración de los elementos externos del entorno antes de comenzar la construcción, entendiendo por elementos externos no solo las condiciones naturales sino el carácter de los edificios de alrededor" ${ }^{12}$. No era tan solo una tarea consistente en los trabajos de jardinería a realizar después de haber concebido un edificio, sino que debía ser objeto de proyecto desde el comienzo del mismo.

La representación en occidente confiere especial importancia a la relación entre la luz y las sombras en sus composiciones pictóricas; en Japón, esta dualidad se define como Notan. Consiste en la utilización de planos oscuros y otros más claros, de forma que su composición determine los planos en sombra del objeto representado, sin que aparezcan sombras arrojadas. Esto convierte a las composiciones en elementos pictóricos bidimensionales, planos, carentes de profundidad. Por este motivo, la disposición de la vegetación excediendo los límites del marco de la imagen sirve para establecer una serie de planos superpuestos que dotan de la sensación de levedad propia de las xilografías de Ando Hiroshige ${ }^{13}$, al tiempo que acentúan la sensación de vista en perspectiva introduciendo al espectador en el dibujo [5].

La composición tipo del dibujo propuesto por Mahony y Griffin se organiza en un panel de tamaño único, de $45 \mathrm{~cm} \times 90 \mathrm{~cm}$, concebido como un grabado en color satinado acabado en tinta o en acuarelas transparentes mezcladas con pegamento, en el que se muestra una visión completa del proyecto ${ }^{14}$. Así, la mitad superior del dibujo se destinaba a una perspectiva exterior del mismo, en la que la vegetación envolvía al edificio. La parte inferior, dividida a su vez en dos franjas horizontales, dedicaba una mitad a las plantas, y la otra mitad a los alzados y las secciones, así como otros elementos singulares. La vegetación se representaba en planta con precisión a fin de significar el carácter de los espacios que rodeaban al edificio principal. De igual manera, en la perspectiva, los elementos naturales, como los árboles o las plantas y flores, parecen romper los límites de las partes en las que estaba dividida la composición para dotar de continuidad y distintas profundidades al conjunto.

Las técnicas del grafismo nipón aplicadas a la presentación de proyectos arquitectónicos resulta una variación significativa con respecto a la tradición Beaux Arts en la que ambos se formaron. Sin embargo, el grado de abstracción con el que las perspectivas exteriores son concebidas consigue dotar al documento, en el que se muestran conjuntamente las plantas y las secciones, de una sensación de extrañamiento que resulta atractiva. Si bien tratan de representar una perspectiva de un objeto final construido inserto entre la vegetación, la observación de sus dibujos permite descubrir cómo el método utilizado no es un sistema meramente descriptivo. Se trata de una invitación al espectador para que imagine un lugar como el presentado pero convertido en realidad. La capacidad evocadora del dibujo se convierte, gracias a este método de representación oriental, en un mecanismo de expresión propio de estos arquitectos. Capaz al mismo tiempo de mostrar un entorno vegetal imaginado en el que se insertará una construcción, de tal manera que esta parece haber sido diseñada para ocupar ese lugar y no otro, una vez se convierta en realidad.

\section{El carácter de la representación oriental como herramienta de proyecto}

Tras la etapa formativa junto a Wright, establecen su práctica profesional de manera autónoma trabajando en diversos proyectos en los Estados Unidos. Uno de ellos es la casa J. G. Melson [6], dentro del desarrollo urbanístico planeado por ambos en Mason City ${ }^{15}$. El plano de situación del lugar, y la lámina que preparan para explicar el proyecto, reflejan el interés por el entorno, por la vegetación y por el agua a los pies de la edificación. La composición del dibujo responde a la organización vertical tipo, mostrando la perspectiva exterior de la vivienda rodeada de vegetación en la parte superior. La representación del reflejo de esta sobre el agua cercana, mediante
${ }^{6}$ MORSE, Edward. Japanese Homes and their surroundings. Boston: Ticknor and Company, 1886

${ }^{7}$ DOW, Arthur Wesley. Composition. A series of exercises selected from a new system of education. Nueva York: The Baker and Taylor company, 1899.

[4] Perspectiva de la casa K. C. De Rodhes. Dibujo de Marion Mahony Griffin. Proyecto de Frank Lloyd Wright. 1906. Fuente: Frank Lloyd Wright Foundation, Scottsdale, Arizona (0602.001).

[5] Ando Hiroshige. Arboleda de Suijin y Masaki. 1856-1858. Fuente: Ashmolean Museum, University of Oxford, UK/Bridgeman Art Library. Annenberg learner.

[6] Casa J. G. Melson. 1912. Fuente: Biblioteca Nacional de Australia.

[5]

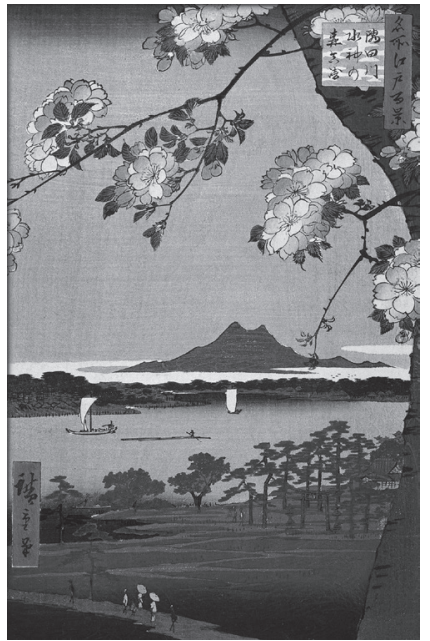

[6]

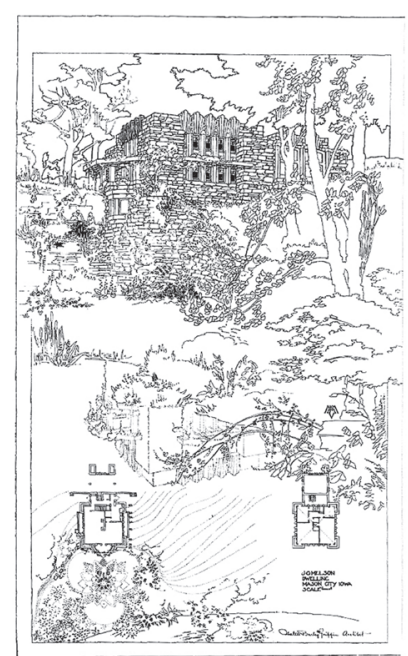


${ }^{8}$ Mahony comienza a trabajar junto a Frank Lloyd Wright en 1895 como encargada de la representación gráfica de los proyectos del estudio. Tras una experiencia previa en las oficinas de Dwight Perkins, en el edificio Steinway Hall de Chicago, se une a la aventura individual de Wright en un periodo de transición y confirmación de un estilo propio transicion y confirmacion de un estlo propio alternativo al aprendido de su maestro Louis Sullivan, en el que las perspectivas exteriores de sus proyectos comienzan a incluir el paisaje y la naturaleza que los rodea, mostrando al espectador la atmósfera de la nueva arquitectura propuesta. Si en su arquitectura se manifiesta una tendencia hacia la simplificación geométrica derivada de lo aprendido del estudio de las estampas japonesas, el método de representación desarrollado por Mahony incorpora la naturaleza como un elemento constructivo más, hasta convertirse en la protagonista de algunas de las escenas como elemento atractor de los espectadores. En 1901 Griffin, tras su etapa como aprendiz también en Steinway Hall, se incorpora al estudio de Wright, como responsable de la dirección de algunos de los proyectos singulares del estudio. A partir de este momento se produce una evolución en su arquitectura, derivada del estudio de las variaciones de la planta centralizada y el empleo de fuertes elementos estructurales ciegos en las esquinas de los edificios. Si bien la significación de macizos y huecos será una constante en la obra de Wright, la verticalidad predominante durante los cinco años en los que Griffin es responsable d deja paso a una arquitectura marcadamente horizontal, especialmente en los últimos ejemplos construidos de las Prairie Houses. En 1906, Griffin abandona el estudio debido a desavenencias económicas y profesionales con Wright. Tres años más tarde Mahony abandona el estudio, tras un periodo convulso en el que las cada vez más frecuentes ausencias de este imposibilitaban un desarrollo ade cuado de los proyectos, uniéndose a Griffin para conformar una asociación que perdurará en el tiempo hasta el fallecimiento prematuro de su socio y marido, en 1937

${ }^{9}$ WRIGHT, Frank Lloyd. The early work of Frank Lloyd Wright. The "Ausgeführte Bauten" of 1911. Nueva York: Dover Publications Inc., 1982.

10 WRIGHT, Frank Lloyd. The Japanese Print: An Interpretation. (consulta 29.03.19) URL: https://babel.hathitrust.org/cgi/pt?id=uc1. c034918470; view=1 up;seq=7. Chicago: The Ralph Fletcher Seymour Co. Fine Arts The Ralph Fletch
Building, 1912.

11 NUTE, Kevin. Frank Lloyd Wright and Japan. The role of traditional Japanese art and architecture in the work of Frank Lloyd Wright. Londres: Routledge, 2000.

12 GRIFFIN, Marion Mahony. "Democratic architecture: its development, its principles and its ideals". Building, vol. 14, $\mathrm{n}^{\circ} 82,12$ Junio 1914, pp. 101-102.

13 La organización de la mayoría de sus dibujos resulta heredera del formato vertical de las xilografías japonesas de Ando Hiroshige. Artista japonés de comienzos del siglo $\mathrm{XIX}$ considerado como uno de los mayores exponentes del paisajismo de su país, sus obras se caracterizan por la representación de escenas naturales asi como urbanas en las que la vegetación es la principal protagonista, (SCHLOMBS, Adele. Hiroshige. Hong Kong: Taschen, 2010). Representante del género conocido como Ukiyo-e, o estampas del mundo flotante, la temática de sus obras responde al concepto budista de la vida entendida como un fenómeno efimero, de manera que se represente en ellas la sensación de levedad. En sus composiciones destaca el uso del color, principalmente el verde, el azul, e dorado y el rojo.

14 GRIFFIN, Marion Mahony. The Magic of America. (consulta 15.05.18) URL: http:// www.artic.edu/magicofamerica/index.html. Chicago: The Art Institute of Chicago, 1949, pp. 286-287. unos trazos punteados, intensifica la presencia de este elemento natural en el conjunto final del proyecto. Además, incluye en la parte inferior del mismo las plantas de la vivienda, así como una serie de motivos vegetales que sirven para dotar al conjunto de unidad. De la utilización de la planta centralizada se pueden descubrir semejanzas con arquitecturas realizadas por Wright en el periodo en el que ambos trabajaron juntos. Los elementos estructurales, situados en las cuatro esquinas, recuerdan a las propuestas para el edificio de oficinas Larkin o el Templo Unitario [7] [8].

Debido a lo singular del enclave natural en el que se iba a desarrollar el proyecto, resultaba más importante mostrar la vivienda significando el carácter único en el que iba a construirse, antes incluso que el espacio interior de la misma. Los mecanismos gráficos utilizados para ocultar el encuentro del nuevo volumen con el suelo o las paredes de piedra existentes mediante otros elementos vegetales, sirve para enfatizar, al igual que en la vista aérea del conjunto, la voluntad de construir una casa enraizada al terreno, no como fruto de una superposición sino como el descubrimiento de un nuevo elemento que emerge de la tierra. El punto de vista elegido, desde el río y en una posición por debajo de las personas que aparecen en un nivel inferior a la izquierda de la composición, significa tanto la compacidad del conjunto como la marcada verticalidad de la arquitectura propuesta en el lugar.

El estudio y devoción por la vegetación local no solo consistía en su dibujo y catalogación, sino que ambos comenzaron a potenciar este respeto por la naturaleza como signo distintivo de su carrera como arquitectos. La utilización y puesta en valor de especies vegetales autóctonas se convirtió en uno de sus mayores retos profesionales al defender su uso, ya que era posible conseguir una mayor belleza natural en el menor tiempo posible y con el menor gasto asumible ${ }^{16}$. Los jardines botánicos de Sydney y de Melbourne fueron lugares en los que ambos descubrieron el potencial que la flora de Australia escondía, y donde conocieron a botánicos y geólogos australianos que les explicaron las características principales del continente en el que ejercían entonces como arquitectos. Desde 1915 y hasta 1940, Mahony realiza una serie de láminas en las que recoge algunas de las especies vegetales más interesantes, a su juicio, del continente australiano, creando una colección de veinticuatro dibujos denominada como Retratos del Bosque, en la que muestra no solo su interés por la naturaleza sino también su dominio de múltiples técnicas de representación basadas en características propias de las estampas japonesas [7] ${ }^{17}$.

Concebidos en primera instancia como ejercicios ajenos a la producción arquitectónica de la pareja, estas láminas resultan una derivación del modelo compositivo tipo utilizado en proyectos previos. Aún así mantienen las proporciones verticales de los lienzos, como signo distintivo de su obra. Esta serie ha de entenderse entonces como un trabajo experimental, donde la naturaleza se convierte en el único elemento de la composición, sin que exista referencia alguna a la escala humana más allá de la que pueda intuirse de la observación de las escenas. Si las geometrías básicas entendidas como principios estructurales fundamentales, según Wright, producen "un encantamiento basado en su carácter simbólico fundado sobre una base matemática" 18; las composiciones de Mahony y Griffin utilizan la representación de los elementos naturales como atractores del espectador mediante una sucesión de planos compuestos por diferentes especies vegetales. Filtros que recuerdan a la sucesión de espacios interiores que configuran los elementos móviles que delimitan las estancias de la vivienda tradicional japonesa.

El empleo de las siluetas para potenciar los efectos de las perspectivas se combina con el uso de diferentes texturas y colores de la vegetación en el entorno natural. La introducción de estos elementos produce una ligera alteración de la percepción de la escala del conjunto, incidiendo en valores tales como la serenidad y libertad propias de un continente por descubrir. La técnica nipona del Ukiyo-e parece servir a su autora para mostrar la sensación de sorpresa y admiración por el paisaje que observa, al tiempo que ensalza la levedad y la condición efímera del hombre en relación con su entorno. Se trata de un reconocimiento de la naturaleza como elemento a preservar y del que el ser humano forma parte.

Serán estos dibujos los documentos que servirán a ambos arquitectos como presentación para introducir la figura del arquitecto del paisaje, desconocida hasta entonces en Australia, y que debía sustituir a la del jardinero a fin de incluir en cada proyecto no solo las condiciones naturales sino también el carácter de los edificios de alrededor. Su labor ha de ir más allá del cuidado y la catalogación de los elementos naturales visibles y de una descripción detallada de sus características. Es necesario un entendimiento de la naturaleza como un proceso que evoluciona con el tiempo, al que el hombre ha de adaptarse y con el que debe convivir. Su arquitectura del paisaje no trata de imponer un trazado geométrico específico sino de encontrar el orden interno propio del lugar para interpretarlo y potenciarlo. 
Tanteado inicialmente, en su etapa como colaboradores de Wright, y desarrollado durante sus primeras obras de forma independiente, en los documentos gráficos preparados para el proyecto de la comunidad residencial de Castlecrag ${ }^{19}$ el entorno se muestra ante el espectador como el protagonista principal en relación a la presencia de la arquitectura en el dibujo. Mahony y Griffin utilizan uno de los Retratos del Bosque [8] para la promoción de este desarrollo inmobiliario, incidiendo así en la subordinación a la naturaleza que les rodea, tanto de la arquitectura como del hombre. En un segundo plano, aparece una perspectiva de una de las viviendas propuestas y en la esquina inferior izquierda, en un tamaño menor, se hace referencia a la planta de la vivienda. La composición vertical se sirve de la vegetación para organizar el papel de forma que enmarca y dirige la visión, por un lado hacia la vivienda propuesta, y por otro hacia la bahía. La perspectiva exterior transforma el marco del dibujo en una masa vegetal de la que no se puede saber el comienzo ni el final, consiguiendo así el efecto de introducir al espectador en la escena, enmarcando la vivienda. Esta dificultad por establecer los límites de la perspectiva incrementa la sensación de libertad que transmiten sus dibujos. El aspecto de naturaleza no domesticada refuerza su idea de subordinación de la arquitectura al paisaje.

Esta presentación del proyecto ante los posibles compradores respondía al deseo de ambos de hacer conscientes a los futuros residentes de que no iban a adquirir simplemente una propiedad en forma de vivienda, sino también un entorno natural del que debían hacerse responsables. De esta manera conseguirían dar a cada uno de los habitantes la oportunidad de alcanzar su sueño. "Quiero que Castlecrag se construya de forma que cada individuo pueda sentir que todo el paisaje es suyo. Sin barreras, sin fronteras" ${ }^{20}$. Aplicando tan solo las operaciones mínimas necesarias sobre el terreno, y sin modificar el entorno natural, la capacidad de adaptación de la arquitectura propuesta queda ligada al pensamiento oriental de tal manera que lo uno no pueda ser concebido sin lo otro. Así la observación de las edificaciones recordará a la totalidad del entorno natural en el que se insertan y viceversa, la lectura del carácter del lugar será completa observando su flora local y entendiendo las construcciones propuestas como parte del mismo.

El proceso de eliminación de lo superfluo en favor de una arquitectura esencial se traduce en una construcción más compacta en la que el empleo de la piedra local y el uso de cubiertas planas convierten las viviendas propuestas en esta comunidad residencial en rocas que emergen del terreno, horadadas de forma puntual para dejar pasar la luz al interior y poder ser habitadas. Un desarrollo dirigido hacia una paulatina reducción del tamaño de cada una de las unidades, simplificando la distribución interior en busca de un modelo habitacional que pudiera adaptarse a las necesidades económicas de diferentes estratos sociales, generando un modelo inclusivo en un entorno natural privilegiado. Un sistema derivado de las viviendas de bajo coste experimentadas junto con Wright tras la llegada en 1906 de Griffin al estudio de este [9], que pueden ser consideradas como el germen de la arquitectura planteada en Castlecrag. La organización en $L$ de la planta baja, de forma que el acceso principal se sitúa junto a la cocina, conectada a su vez con el comedor y el salón principal ocupando la mitad de la superficie disponible, será una solución recurrente de gran parte de las viviendas desarrolladas a las afueras de Sydney entre 1920 y 1935 [10]. La situación de los elementos de servicio y el acceso a las viviendas en la parte más próxima a las avenidas principales, permite colocar la zona del salón y el comedor orientados hacia las vistas de la vegetación y la bahía cercana. Esto convierte a la naturaleza en la protagonista desde el interior de las casas, extendiendo así los límites construidos hasta considerar el entorno en el que se insertan como una estancia más al aire libre.

[7]

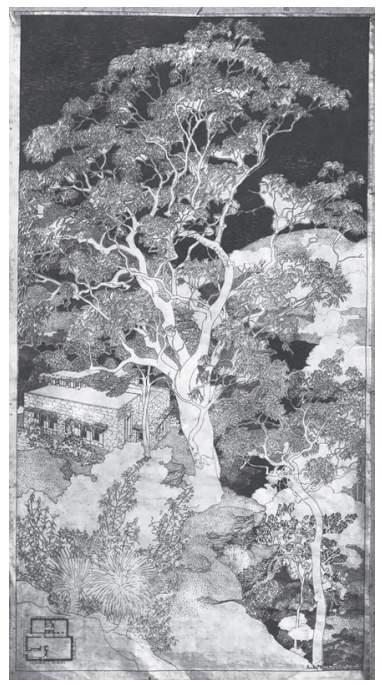

[8]

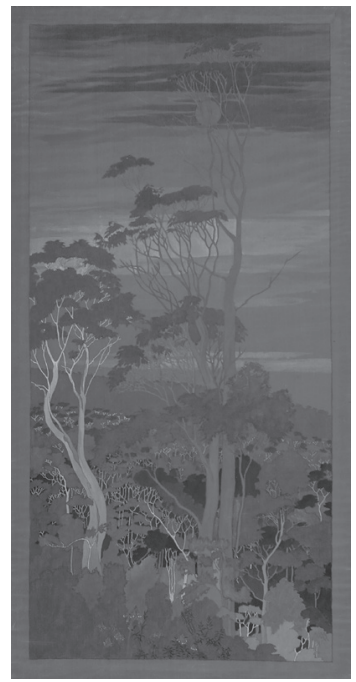

[7] Retratos del bosque. Título de la imagen: Eucalyptus Urnigera Tasmania/Scarlet Bark, 1919. Fuente: Mary and Leigh Block Museum of Art

\section{[8] Retratos del bosque. Título de la imagen: $N^{\circ} 6$} Angophora Lanceolatal A Castlecrag Home in a Castlecrag Gully. 1920. Fuente: Biblioteca Nacional de Australia.
${ }^{15}$ La crítica al modelo residencial suburbano en los Estados Unidos a comienzos del siglo XX, fundamentada en la creciente segregación social derivada de las desigualdades económicas provocadas por la irrupción de la máquina en el sistema productivo del pais, sirve como argumento para el desarrollo de una alternativa en la que se combinará la propiedad privada con la cesión y gestión de parte de los terrenos de la agrupación como espacios comunitarios. Afines al modelo económico planteado por Henry George, en el que propone un sistema impositivo que afecte únicamente al valor del suelo en el que se construye (George, Henry. The writtings of Henry George. Progress and poverty. Nueva York: Doubleday \& McClure, 1898); $y$ a las alternativas de ordenación social y urbana como la planteada por Patrick Geddes y su movimiento civico (Hysler-Rubin, Noah. Patrick Geddes and Town Planning. A critical view. Londres y Nueva York: Routledge. Taylor \& Francis Group, 2011); Mahony y Griffin plantean una solución alternativa denominada comunidades domésticas. Mahony y Griffin desarrollan entre 1911 y 1912 los proyectos para las agrupaciones residenciales de Trier Center Neighborhood, en Winnetka, Illionois, Ridge Quadrangles y Emory Hills, en Chicago, Clark's Resubdivision en Grinell, lowa, e lalia, en Lee County, Florida, desde el planeamiento urbano como herramienta capaz de establecer las condiciones básicas, no solo para la distribución de usos y viarios, sino para la gestión administrativa de la titularidad de la propiedad privada y pública del suelo. La relación entre vivienda y paisaje se combina con la definición de espacios verdes y zonas deportivas o culturales que potencien la sensación de comunidad. Estas comunidades servirán para testar la solución propuesta en 1912 en Rock Crest / Rock Glen, en Mason City, lowa como un proyecto previo al de Castlecrag, ya en Australia, considerado como la confirmación de las comunidades residenciales cooperativas como una alternativa viable. En ella combinan el modelo económico capitalista y el socialista, promoviendo el beneficio individual dentro de una visión global de la comunidad. Al igual que el sistema de representación oriental, cada parte significada como singular, pero dependiente de un conjunto de orden superio identificado con la naturaleza

16 GRIFFIN, Marion Mahony. The Magic of America. (consulta 15.05.18) URL: http:// www.artic.edu/magicofamerica/index.html. Chicago: The Art Institute of Chicago, 1949, p. 122.

${ }^{17}$ La mayoría de estos dibujos se realizaban como bocetos en grafito que posteriormente se terminaban a tinta sobre un papel traslúcido ligeramente satinado. De ahi, el dibujo se transfería a una tela de seda sobre la que se transferia a una tela de seda sobre la que se
aplicaba color mediante diferentes tintes. La transferencia del papel a la seda se realizaba mediante un proceso de reproducción similar a las utilizadas en la fotografía de entonces con los cianotipos. Tras este paso, los dibujos se terminaban aplicando color con tintas similares a las de las xilografias japonesas. Las zonas en las que no se coloreaba dejaban a la vista el tejido brillante de la seda como base del dibujo, que aportaba unos brillos adicionales a los generados por los tintes. (WOOD Deborah. Marion Mahony Griffin. Drawing th Form of Nature. Evanston, Illinois: Mary and Leigh Block Museum of Art, 2005, p.11).

18 WRIGHT, Frank Lloyd. The Japanese Print An Interpretation. (consulta 29.03.19) URL: https://babel.hathitrust.org/cgi/pt?id=uc1. c034918470;view=1 up;seq=7. Chicago: The Ralph Fletcher Seymour Co. Fine Arts Building, 1912, p.15. 
${ }^{19}$ Situada a las afueras de las ciudad de Sydney, en la bahía de Middle Harbour, la comunidad doméstica de Castlecrag se desarrolla entre los años 1920 y 1935, basada en el modelo iniciado en los Estados Unidos, en el que los propietarios eran a su vez los gestores de los espacios públicos comunitarios. En esta ocasión Mahony y Griffin ejercen el papel de promotores, arquitectos y propietarios, al crear en 1919 una sociedad denominada GSDA (Global Sydney Development Association Ltd.) en la que junto a otros inversores deciden adquirir los terrenos para construir un modelo residencial alternativo, así como una arquitectura atendiendo a las condiciones especificas del lugar. Durante los quince años en los que continúan desarrollando el proyecto, como residentes y miembros de la comunidad, realizan cuarenta y seis proyectos de viviendas de los cuales finalmente se construyen diecisiete. En LESLIE, Esther. The suburb of Castlec rag. A community history. Sydney: Willoughby Municipal Council, 1988, se detalla el proceso de creación de esta comunidad y se analizan algunas de sus viviendas más representativas, como la casa Fishwick (URL: http://www. fishwickhouse.org), ofreciendo a su vez una visión actual de la comunidad, y cómo ha evolucionado desde su creación. Para más información visitar la web oficial de Castlecrag en URL: https://castlecrag.org.au.

${ }^{20}$ GRIFFIN, Marion Mahony. The Magic of America. (consulta 15.05.18) URL: http:// www.artic.edu/magicofamerica/index.html. Chicago: The Art Institute of Chicago, 1949, p. 526.

[9] A Fireproof House for $\$ 5,000$. Frank Lloyd Wright. Fuente: The Ladies Home Journal, April 1907, p. 24.

[10] Planta casa Guy. Castlecrag. 1925 Fuente: Walter Burley Griffin Society Inc. Collection, imagen de Bob Meyer.

\section{Conclusiones}

Las palabras de Griffin en las que se valora el sistema de representación japonés como aquel capaz de extraer lo esencial obviando lo irrelevante, pueden entenderse ahora como una herramienta desde la que generan sus proyectos, atendiendo a todas sus escalas, desde lo urbano a la construcción final de los mismos. La precisión con la que el entorno natural es dibujado les permite descubrir relaciones entre la naturaleza y la arquitectura, así como potenciar espacios al aire libre en los que el individuo pueda valorar su entorno cercano, al tiempo que es consciente de formar parte de un todo. La búsqueda de una tipología en planta a partir del cuadrado como geometría básica, cuya subdivisión a su vez en salas de menor tamaño genera estancias interiores, retoma los principios compositivos empleados en el Ukiyo-e, y ensalzados por Wright tras su análisis en busca de un lenguaje universal para la arquitectura.

El grafismo utilizado para mostrar sus ideas responde no solo a unas técnicas determinadas, sino a la voluntad de condensar en cada una de estas representaciones los conceptos fundamentales de sus proyectos. La significación del entorno en el que construir, así como la relación de dependencia entre la arquitectura y la naturaleza, enfatizan la responsabilidad de preservación, coexistencia y puesta en valor de estas por parte del usuario. La abstracción geométrica y constructiva hacia la que evoluciona su arquitectura debe entenderse como una actitud en busca de una belleza universal basada en conceptos orientales, así como un manifiesto en favor de un uso responsable de los recursos naturales con los que el individuo debe convivir, desde su condición efímera en el mundo natural en el que habita.
$[10]$

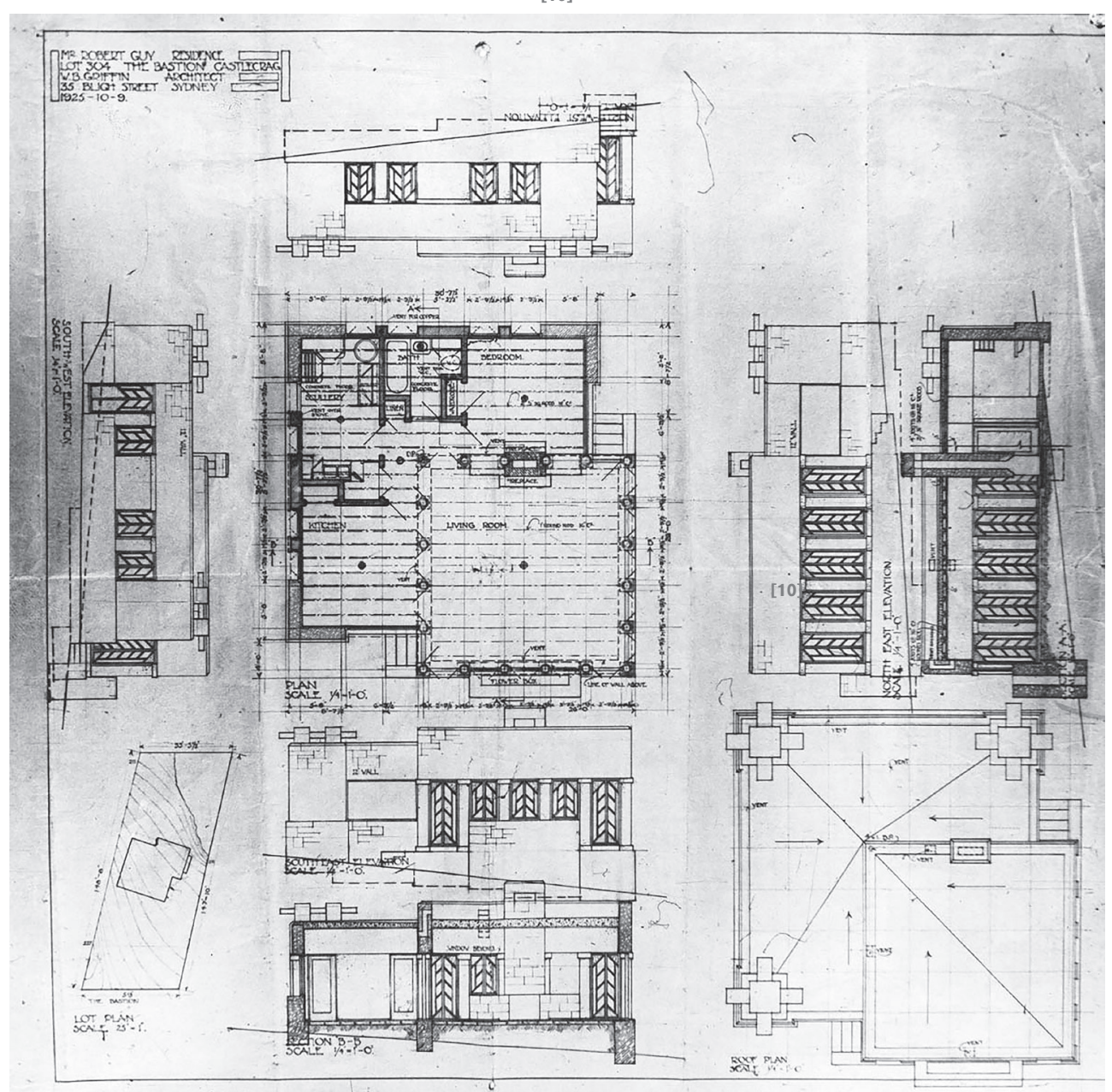




\section{3 | El Japonismo en Marion Mahony Griffin y Walter Burley Griffin. De los conceptos gráficos del Ukiyo-e hacia una estrategia proyectual alternativa _Javier \\ Mosquera González}

La significación de la naturaleza, como referente desde el que Marion Mahony Griffin y Walter Burley Griffin tratan de generar una arquitectura alternativa, deviene de las profundas convicciones de ambos en el ser humano como aquel cuya relación directa con el entorno en el que habita produce una unidad indisoluble. Herederos del pensamiento trascendentalista americano, el interés de ambos por la cultura oriental, y en concreto por el sistema de representación nipón Ukiyo-e, les sirve para desarrollar estrategias proyectuales que condensan conceptos propios de esta disciplina artística, reflejando las características esenciales de la naturaleza no solo de forma gráfica sino también como realidad construida. Los proyectos para las comunidades residenciales de Rock Crest / Rock Glen y Castlecrag, en los Estados Unidos y Australia, son concebidos a partir de las ideas contenidas en los métodos gráficos nipones. El carácter efímero del individuo, en relación con el lugar en el que habita, implica una subordinación de este ante las leyes naturales que lo definen desde la escala urbana, y su implantación en el lugar, hasta la construcción y organización interior de cada una de las viviendas.
13 Japanism in Marion Mahony Griffin and Walter Burley Griffin. From Ukiyo-e graphical concepts to an alternative design strategy_Javier Mosquera González

The significance of nature as a reference from which Marion Mahony Griffin and Walter Burley Griffin try to generate an alternative architecture, comes from the deep convictions of both in the human being as one whose direct relationship with the environment in which they live produces a unity indissoluble. Heirs of the American transcendentalism thought, the interest of both for the oriental culture, and in particular for the Japanese representation system Ukiyo-e, serves to develop project strategies that condense concepts of this artistic discipline, reflecting the essential characteristics of nature not only graphically but also as constructed reality. The projects for the residential communities of Rock Crest / Rock Glen and Castlecrag, in the United States and Australia, are conceived from the ideas contained in the Japanese drawing style. The ephemeral nature of the individual in relation to the place where he lives, implies a subordination of the latter to the natural laws that define it, from the urban scale and its implantation in place, to the construction and internal organization of each one of the households.

\section{3 | El Japonismo en Marion Mahony Griffin y Walter Burley Griffin. De los conceptos gráficos del Ukiyo-e hacia una estrategia proyectual alternativa_Javier Mosquera González}

BANERJ, Shiben. Inhabiting the world: Architecture, Urbanism, and the Global MoralPolitics of Marion Mahony and Walter Burley Griffin. Tesis doctoral. Director: Arindam Dutta. Massachusets Institute of Technology MIT, 2015.

BIRRELL, James. Walter Burley Griffin, Brisbane: University of Queensland Press, 1964.

CARPENTER, Edward. Civilization: Its Cause and Cure, and Other Essays. Londres: G. Allen \& Unwin, 1914.

DOW, Arthur Wesley. Composition. A series of exercises selected from a new system of education. Nueva York: The Baker and Taylor company, 1899.

GEORGE, Henry. The writtings of Henry George. Progress and poverty. Nueva York: Doubleday \& McClure, 1898.

GRIFFIN, Dustin. The writtings of Walter Burley Griffin. Nueva York: Cambridge University Press, 2008.

GRIFFIN, Marion Mahony. "Democratic architecture: its development, its principles and its ideals". Building, vol. 14, $\mathrm{n}^{\circ} 82,12$ (Junio 1914).

GRIFFIN, Marion Mahony. The Magic of America. (Consulta el 15 de mayo de 2018) URL: http://www.artic.edu/magicofamerica/index.html. Chicago: The Art Institute of Chicago, 1949.

GRIFFIN, Walter Burley. "Architecture in American Universities", Journal of Proceedings of Royal Victorian Institute of Architects, septiembre 1913.

HARRISON, Peter. Walter Burley Griffin: Landscape Architect. Canberra: National Library of Australia, 1995.

HYSLER-RUBIN, Noah. Patrick Geddes and Town Planning. A critical view. Londres y Nueva York: Routledge. Taylor \& Francis Group, 2011.

JOHNSON, Donald Leslie, The Architecture of Walter Burley Griffin. South Melbourne: Macmillan Company of Australia, 1977.

LARSON, Paul. "Marion Mahony \& Walter Burley Griffin: The marriage of drawing and architecture". The Print Collector's Newsletter, Vol. $13, \mathrm{n}^{\circ} 2$, mayo-Junio 1982.

LESLIE, Esther. The suburb of Castlecrag. A community history. Sydney: Willoughby Municipal Council, 1988.

McGREGOR, Alasdair. Grand Obsessions: The life and work of Walter Burley Griffin and Marion Mahony Griffin. Londres: Lantern, 2009.

MORSE, Edward. Japanese Homes and their surroundings. Boston: Ticknor and Company, 1886.

NUTE, Kevin. Frank Lloyd Wright and Japan. The role of traditional Japanese art and architecture in the work of Frank Lloyd Wright. Londres: Routledge, 2000.

PEISCH, Mark. The Chicago School of Architecture: Early Followers of Sullivan and Wright. Nueva York: Random House, 1964.

ROBERTS, Ellen. "Ukiyo-e in Chicago: Frank Lloyd Wright, Marion Mahony Griffin and the Prairie School". Art in Print, Vol. 3, N 2, julio-agosto 2013.

SCHLOMBS, Adele. Hiroshige. Hong Kong: Taschen, 2010.

SPATHOPOULOS, Wanda. The Crag. Castlecrag 1924-1938. New South Wales: Brandl \& Schlesinger, 2007.

TINCH, Rebecca. Locating Modernity: Japonisme, Gender, and Enchantment at the 1893 World's Fair. Tesis fin de Máster. Directora: Mary E. Frederickson. Miami University, 2012.

V.V.A.A. Beyond Architecture. Marion Mahony Griffin and Walter Burley Griffin. America. Australia. India. Sydney: Powerhouse publishing, 1998.

V.V.A.A. Building For Nature. Walter Burley Griffin and Castlecrag. New South Wales: Walter Burley Griffin Society, 1994.

V.V.A.A. Japonismo. La fascinación por el arte japonés. Barcelona: Obra Social La Caixa, 2013.

V.V.A.A. La estampa japonesa. Frank Lloyd Wright. Vitoria-Gasteiz: Sans Solei, 2018.

V.V.A.A. Rock Crest / Rock Glen. Mason City, lowa. The American Masterwork of Marion M. and Walter B. Griffin. Chicago: Walter Burley Griffin Society of America, 2014.

WRIGHT, Frank Lloyd. An Autobiography. New York: Duell, Sloan and Pearce, 1943.

WRIGHT, Frank Lloyd. The early work of Frank Lloyd Wright. The "Ausgeführte Bauten" of 1911. Nueva York: Dover Publications Inc., 1982.

WRIGHT, Frank Lloyd. The Japanese Print: An Interpretation. (Consulta el 29 de marzo de 2019) URL: https://babel.hathitrust.org/cgi/pt?id=uc1. c034918470;view=1 up;seq=7. Chicago: The Ralph Fletcher Seymour Co. Fine Arts Building, 1912.

WRIGHT, Frank Lloyd. Wasmuth Portfolio. (Consulta el 27 de marzo de19) URL: https://collections.lib.utah.edu/details?id=204451

WOOD, Deborah. Marion Mahony Griffin. Drawing the Form of Nature. Evanston, Illinois: Mary and Leigh Block Museum of Art, 2005. 